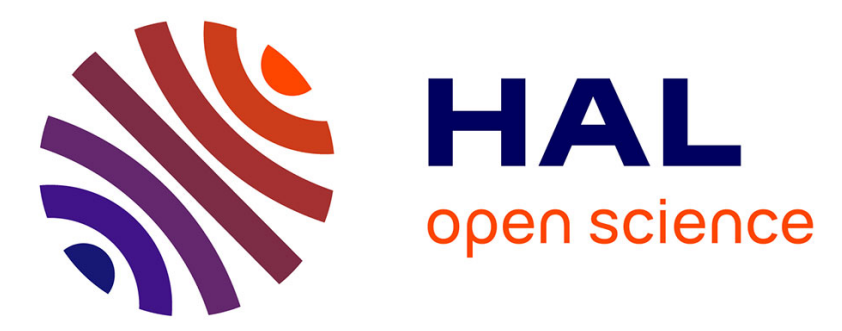

\title{
Modélisation de faisceaux de transducteurs ultrasonores par spectre angulaire impulsionnel
}

\author{
J. de Belleval, N. Messaoud Nacer, N. Mercier
}

\section{To cite this version:}

J. de Belleval, N. Messaoud Nacer, N. Mercier. Modélisation de faisceaux de transducteurs ultrasonores par spectre angulaire impulsionnel. Journal de Physique IV Proceedings, 1994, 04 (C5), pp.C5-311C5-314. 10.1051/jp4:1994563 . jpa-00253058

\section{HAL Id: jpa-00253058 https://hal.science/jpa-00253058}

Submitted on 1 Jan 1994

HAL is a multi-disciplinary open access archive for the deposit and dissemination of scientific research documents, whether they are published or not. The documents may come from teaching and research institutions in France or abroad, or from public or private research centers.
L'archive ouverte pluridisciplinaire HAL, est destinée au dépôt et à la diffusion de documents scientifiques de niveau recherche, publiés ou non, émanant des établissements d'enseignement et de recherche français ou étrangers, des laboratoires publics ou privés. 


\title{
Modélisation de faisceaux de transducteurs ultrasonores par spectre angulaire impulsionnel
}

\author{
J.F. DE BELLEVAL, N. MESSAOUD NACER et N. MERCIER
}

LG2mS, URA 1505 du CNRS, Université de Technologie de Compiègne, BP. 649, 60206 Compiègne cedex, France

\begin{abstract}
In the scope of ultrasonic non destructive testing, the knowledge of the field of the transducer in the material to be tested is essential to locate the detected flaw and moreover to evaluate them in a precise way. We have developed a model based upon a decomposition into plane waves (angular spectrum), but while this is classically achieved for monochromatic fields, we have extended the formalism to transient domain. The first steps of the calculation are classical ; from the knowledge of the transient field into a plane, a time Fourier transform permits the decompostion into monochromatic waves, then a spatial Fourier transform gives the decomposition into plane waves. Then it is possible to obtain the transient angular spectrum by mean of a change of variable and a time recomposition. The recomposition an an point of the field is then no more than a summation, with suitable delays, of all these plane waves. The advantage of this method is that the decomposition into transient plane waves (the longest operation) has to be performed only once for a given transducer, since the fit to arbitrary geometrical configurations (interface, incidence angle,...) is taken into account only at the step of the final recomposition.
\end{abstract}

\section{INTRODUCTION}

En évaluation non destructive de défauts par ultrasons, la connaissance du champ des transducteurs dans les matériaux testés est essentielle pour localiser les défauts détectés et plus encore pour les évaluer de façon précise. Un grand nombre de méthodes de prévision de ces champs ont été développées soit à partir de modèles analytiques soit numériquement. Les modèles analytiques sont généralement plus faciles à manier mais s'adaptent moins aux particularités des transducteurs réels. Les premières approches se sont souvent limitées à une représentation monochromatique, nous avons ainsi développé un modèle basé $[1,2]$ sur une décomposition en ondes planes monochromatiques du champ ultrasonore. Ce modèle utilise une Transformation de Fourier Rapide (FFT) et permet la prévision d'un champ ultrasonore qu'il suffit de définir dans un plan soit analytiquement soit à partir de données expérimentales. Il prend en compte la traversée d'interfaces planes ou non planes. En pratique les transducteurs utilisés en évaluation non destructive ont des bandes passantes de plus en plus grandes, la représentation monochromatique ne suffit alors plus. Un certain nombre d'approches ont donc été faites, citons celle de Stepanishen [3] qui a l'avantage de la simplicité mais qui est limité au rayonnement d'un piston. Nous proposons l'extension de notre modèle de décomposition en ondes planes avec deux approches, la première classique consiste à étudier le champ avec la méthode précédemment décrite fréquence par fréquence et à recomposer la réponse temporelle en chaque point par transformation de Fourier, la seconde consiste à recomposer la réponse temporelle pour chaque direction de propagation, au niveau du plan de référence. On a ainsi une 
décomposition en ondes planes impulsionnelles. La recomposition en un point quelconque du champ n'est alors plus qu'une sommation avec des retards appropriés de toutes ces ondes planes. L'avantage de cette méthode est que la décomposition en ondes planes impulsionnelles (opération la plus longue) n'est à faire qu'une fois pour un transducteur, l'adaptation à des configurations géométriques diverses (interface, angle d'incidence,...) n'étant prise en compte qu'au moment de la recomposition finale. L'une et l'autre méthode permettent la prise en compte de façon tout à fait rigoureuse de la traversée d'interfaces planes séparant deux milieux différents.

\section{APPROCHE CLASSIQUE}

La première méthode, déduite d'un formalisme développé initialement en optique [Goodman], est basée sur la décomposition en ondes planes d'un faisceau monochromatique. Cette décomposition est obtenue par une simple transformation de Fourier si l'onde est connue dans un plan. La propagation de chacune de ces ondes puis une recomposition permettent de déduire le champ dans un autre plan. Le cas impulsionnel se ramène au cas monochromatique à l'aide d'une décomposition fréquentielle par transformation de Fourier sur la variable temporelle.

Ainsi supposons connu $\varphi_{o}\left(x_{1}, x_{2}, t\right)$ un paramètre caractérisant l'onde (potentiel scalaire, pression, $\ldots$ ) dans un plan que nous appellerons plan de référence (nous prendrons un référentiel dont le plan $\mathrm{x}_{1} O \mathrm{Ox}_{2}$ est confondu avec celui-ci). A l'aide d'une double transformée de Fourier spatiale et d'une transformée de Fourier inverse temporelle, $\varphi_{o}\left(x_{1}, x_{2}, t\right)$ peut s'exprimer comme la somme d'ondes planes monochromatiques de pulsation $w$ et de vecteur nombre d'onde $\mathbf{k}\left(k_{1}, k_{2}, k_{3}\right)$ :

$$
\varphi_{o}\left(x_{1}, x_{2}, t\right)=\iiint \varphi_{0}\left(k_{1}, k_{2}, \omega\right) \exp \left\{i\left(\omega t-k_{1} x_{1}-k_{2} x_{2}\right)\right\} d \omega d k_{1} d k_{2}
$$

avec

$$
\varphi_{o}\left(k_{1}, k_{2}, \omega\right)=\frac{1}{8 \pi^{3}} \iiint \varphi_{o}\left(x_{1}, x_{2}, t\right) \exp \left\{-i\left(\omega t-k_{1} x_{1}-k_{2} x_{2}\right)\right\} d t d x_{1} d x_{2}
$$

L'expression (2) donne le spectre spatio-temporel du faisceau dans le plan de référence, il correspond à l'amplitude complexe des ondes planes monochromatiques composant le faisceau, on l'appelle souvent spectre angulaire. Celles-ci sont caractérisées par leur pulsation $w$ et leur vecteur nombre d'onde $\mathbf{k}$ dont la troisième composante $k_{3}$ est donnée par la relation de dispersion du milieu. S'il est homogène et isotrope de vitesse de propagation $c$, elle s'écrit :

$$
k_{1}^{2}+k_{2}^{2}+k_{3}^{2}=\omega^{2} / c^{2}
$$

$k_{3}$ prend des valeurs réelles ou imaginaires pures selon que l'onde considérée est propagative ou ívansouvitu.

On peut déduire $\varphi$ en tout point au moyen de l'expression :

$$
\varphi\left(x_{1}, x_{2}, x_{3}, t\right)=\iiint \varphi_{o}\left(k_{1}, k_{2}, \omega\right) \exp \left\{i\left(\omega t-k_{1} x_{1}-k_{2} x_{2}\right)-i k_{3} x_{3}\right\} d \omega d k_{1} d k_{2}
$$

A partir de ce formalisme il est possible de reconstruire par les mêmes méthodes que celles que nous avons développées et exposées en [2] le champ acoustique dans un plan parallèle au plan de référence, par un changement de référentiel celui dans un plan quelconque et par l'application des lois de Snell-Descartes le champ réfléchi ou transmis par une interface plane séparant deux milieux quelconques. 
Si les transformées de Fourier sont réalisées numériquement à l'aide de l'algorithme de Transformation de Fourier Rapide par exemple, un certain nombre de précautions sont à prendre notamment sur le pas et le domaine d'échantillonnage.

\section{DECOMPOSITION EN ONDES PLANES IMPULSIONNELLES}

Exprimons maintenant la formule (1) à l'aide d'une autre variable que le vecteur nombre d'onde, soit le vecteur lenteur qui s'écrit :

$$
\mathbf{m}=\mathbf{k} / \mathbf{w}=\mathbf{n} / \mathfrak{c}
$$

où $\mathbf{n}$ est le vecteur unitaire caractérisant la direction de propagation de l'onde plane considérée.

La formule (1) s'écrit alors :

$$
\begin{aligned}
\varphi_{o}\left(x_{1}, x_{2}, t\right) & =\iiint \varphi_{o}\left(\omega m_{1}, \omega m_{2}, \omega\right) \exp \left\{i \omega\left(t-m_{1} x_{1}-m_{2} x_{2}\right)\right\} \omega^{2} d \omega d m_{1} d m_{2} \\
& =\iint \phi_{0}\left(m_{1}, m_{2}, t\right) \exp \left\{-i \omega\left(m_{1} x_{1}+m_{2} x_{2}\right)\right\} d m_{1} d m_{2}
\end{aligned}
$$

avec

$$
\phi_{0}\left(m_{1}, m_{2}, t\right)=\int \varphi_{0}\left(\omega m_{1}, \omega m_{2}, \omega\right) \exp (i \omega t) \omega^{2} d \omega
$$

L'expression (6) représente la décomposition du faisceau dans le plan de référence en ondes planes impulsionnelles de direction de propagation, la direction du vecteur lenteur $\mathbf{m}$. Par analogie avec le spectre angulaire monochromatique, nous appellerons l'expression (7) spectre angulaire impulsionnel. D'une manière analogue à celle du premier formalisme on peut déduire l'expression de $\varphi$ en un point quelconque de l'espace, l'expression s'écrit :

$$
\begin{aligned}
\varphi_{o}\left(x_{1}, x_{2}, x_{3}, t\right) & =\iiint \varphi_{o}\left(\omega m_{1}, \omega m_{2}, \omega\right) \exp \left\{i \omega\left(t-m_{1} x_{1}-m_{2} x_{2}-m_{3} x_{3}\right)\right\} \omega^{2} d \omega d m_{1} d m_{2} \\
& =\iint \phi_{o}\left(m_{1}, m_{2}, t-m_{1} x_{1}-m_{2} x_{2}-m_{3} x_{3}\right) d m_{1} d m_{2}
\end{aligned}
$$

Nous constatons que l'expression (8) est la sommation de toutes les ondes planes impulsionnelles prises à un temps retardé de la quantité qu'il faut pour franchir la distance qui sépare l'origine du référentiel au plan d'onde passant par le point d'observation. Autrement dit le retard à prendre en compte est celui qui permet à un plan d'onde passant par l'origine de se propager jusqu'à rencontrer le point d'observation (figure 1). Ces retards se calculent aisément à partir de considérations géométriques élémentaires.

Dans le cas où le faisceau interagit avec une interface plane, le même principe continue à s'appliquer puisque les lois de Snell-Descartes nous donnent les changements de direction des ondes planes ainsi que les coefficients de transmission et de réflexion. Ces coefficients étant indépendants de la fréquence les mêmes lois s'appliquent aux ondes impulsionnelles. La figure 2 présente la construction géométrique correspondant à ce cas qui permet de déduire simplement les formules qui en découlent.

L'avantage de cette méthode est que l'opération la plus longue du calcul n'est à faire qu'une fois pour un transducteur donné, c'est la décomposition en ondes planes impulsionnelles. Cette partie nécessite dans le cas tridimensionnel une triple transformée de Fourier suivie après un changement de variable d'une transformée inverse. Il suffit ensuite de faire des sommations des formes d'ondes temporelles obtenues pour chaque direction $\mathbf{m}$, après avoir fait un décalage temporel qui seul dépend de la géométrie étudiée (angle d'incidence, position du ou des points d'observation, ...). 


\section{CONCLUSION}

Nous avons développé une méthode de modélisation du champ de transducteurs ultrasonores dans un milieu quelconque et pouvant prendre en compte la traversée d'interface plane séparant deux milieux. Ce modèle prend en compte l'aspect impulsionnel du champ à l'aide d'une extension de la notion de spectre angulaire. Nous avons en effet montré qu'un changement de variable (composantes du vecteur lenteur au lieu de celles du vecteur nombre d'onde) au niveau du spectre spatio-temporel du champ permet de recomposer la réponse temporelle pour chaque direction de propagation. Nous avons ainsi une décomposition en ondes planes impulsionnelles (spectre angulaire impulsionnel). La recomposition en un point quelconque du champ n'est alors plus qu'une simple sommation avec des retards appropriés de toutes ces ondes planes. L'avantage de cette méthode est que la décomposition en ondes planes impulsionnelles (opération la plus longue) n'est à faire qu'une fois pour un transducteur, l'adaptation à des configurations géométriques diverses (interface, angle d'incidence,...) n'étant prise en compte qu'au moment de la recomposition finale.

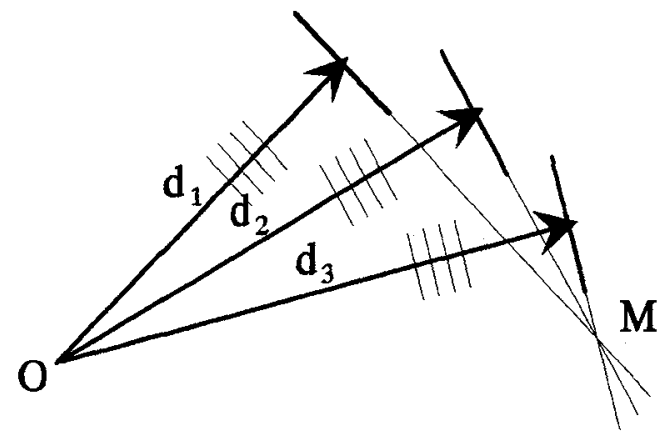

Fig. 1 Sommation au point $\mathrm{M}$ de toutes les ondes planes impulsionnelles avec un retard $\mathrm{t}_{\mathbf{i}}$ égal à $\mathrm{d}_{\mathbf{i}} / c$.

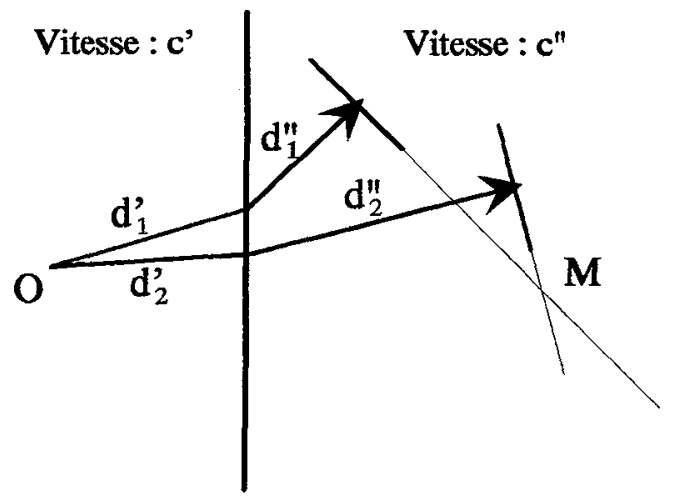

Fig. 2 Interaction avec une interface plane, cas de la transmission, le retard s'écrit $\mathrm{t}_{\mathrm{i}}=\mathrm{d}_{\mathrm{i}}^{\prime \prime} / c^{\prime}+\mathrm{d}^{\prime \prime}{ }_{\mathrm{i}} / \mathcal{c}^{\prime \prime}$

\section{REFERENCES}

[1] Souissi A. de Belleval J.F. Gatignol Ph.

Ultrasonic field of a transducer behind a plane fluid solid interface

Prog. Quant. NDE, Thomson D.O. and Chimenti D.E. ed., Vol 8, Plenum Press 1989

[2] de Belleval J.F. Souissi A. Gatignol Ph. Mercier N.

Modélisation numérique du champ acoustique de transducteurs en présence d'interfaces

quelconques ou de milieux multicouches.

Colloque de Physique C3, suppl J. Phys Tome 51, No 17, (1990).

[3] Stepanishen P.R.

Transient radiation from pistons in an infinite planar baffle

J. Acoust. Soc. Am., Vol 49 (5), 1629-1638, (1971). 